Enfoques. Revista de Investigación en Ciencias de la Administración http://doi.org/10.33996/revistaenfoques.v3i10.61 No. 10 | Volumen 3 | Abril - Junio 2019 http://revistaenfoques.org ISSN: $2016-8219$ pp. $135-148$

\section{GESTIÓN DE PROYECTOS DE INVERSIÓN DE CAPITAL EN LA INDUSTRIA PETROLERA DE LA COSTA ORIENTAL DEL LAGO DEL ESTADO ZULIA}

\author{
PROJECT MANAGEMENT CAPITAL INVESTMENT IN THE OIL \\ INDUSTRY OF THE EAST COAST OF LAKE ZULIA STATE
}

\author{
Daiber Enrique Gómez Güerere
}

Artículo recibido enero 2019 | Arbitrado febrero 2019| Publicado 01 de abril 2019

\section{Resumen}

El presente artículo tiene finalidad analizar la gestión de proyectos de inversión de capital en la industria petrolera de la Costa Oriental del Lago del estado Zulia. Metodológicamente se tipificó como descriptiva, con diseño no experimental transeccional de campo. Para la recolección de los datos se aplicó la técnica de la encuesta y como instrumento un cuestionario de alternativas múltiples, el mismo fue validado por cinco (05) expertos en el área de proyectos y metodología de la investigación. Dentro de las dimensiones estudiadas están: las fases de visualización, conceptualización, definición, implantación y operación. Finalmente se concluyó, que existe un moderado cumplimiento en la gestión de proyectos de inversión en la industria petrolera, lo que podría dar significado a los constantes problemas en los proyectos que allí se presentan generando consigo altos costos adicionales a los estimados, demora excesiva en la entrega de las instalaciones o equipos, personal no capacitado para el área a trabajar, baja calidad en los procesos productivos.

Palabras clave: Fases de un proyecto; gestión de proyectos; proyectos de inversión de capital

\begin{abstract}
This article aims to analyze the management of capital investment projects in the oil industry of the East Coast of Lake Zulia state. Methodologically it was typified as descriptive, with non-experimental field transectional design. For the data collection, the survey technique was applied and as an instrument a multiple alternative questionnaire, it was validated by five (05) experts in the area of research projects and methodology. Within the dimensions studied are: the phases of visualization, conceptualization, definition, implementation and operation. Finally, it was concluded that there is a moderate compliance in the management of investment projects in the oil industry, which could give meaning to the constant problems in the projects presented there generating high additional costs to those estimated, excessive delay in the delivery of facilities or equipment, untrained personnel for the area to work, poor quality in production processes.
\end{abstract}

Key words: Capital investment projects; phases of a project; project management
Daiber Enrique Gómez Güerere gdaiber05@gmail.com Universidad del Zulia, Venezuela

Ingeniero Mecánico, Magister en Gerencia de empresas, mención Gerencia de Operaciones

\title{
135
}




\section{INTRODUCCIÓN}

Las organizaciones actualmente se desarrollan en contextos cada vez más dinámicos, los nuevos avances tecnológicos de la era las rodean de entornos competitivos lo que hace cada vez más difícil su estabilidad en el mercado, ya que no solo deben satisfacer las necesidades del cliente, sino también superar las expectativas que se tienen del producto o servicio que ofrecen. Este escenario lleva a las empresas a buscar métodos y soluciones para mantenerse en el mercado, garantizando su crecimiento y supervivencia dentro del mismo.

$\mathrm{Si}$ bien es cierto que los mercados evolucionan con una rapidez vertiginosa, y la capacidad para adaptarse a esas variaciones es fundamental; las organizaciones se ven en la necesidad de formar una correcta gerencia empresarial, que garantice que sus funciones, ante una competitividad sumamente dinámica, se ejecuten en forma asertiva con el propósito de evolucionar conjuntamente con el mercado e incluso, frente a un mundo moderno y globalizado, poder anticiparse a los cambios con ventajas competitivas.

En ese mismo orden de ideas, Rubio (2008), expresa que el propósito principal de la gerencia empresarial es asegurar que todos los miembros de la organización se integren para formar una empresa común. De ahí que el proceso contemple una correcta información para alcanzar un conjunto de objetivos que busquen dar respuesta a las necesidades que tiene toda empresa; esto a través de la planificación de distintos proyectos aplicados según las necesidades básicas de la corporación.

Desde esta perspectiva, un proyecto dentro de la empresa es una tarea compleja, precisamente porque se trata de algo inexistente, algo que toma forma en la medida que se van realizando las tareas asignadas para dar respuesta a las solicitudes planteadas. Según PMBOK (2004), los proyectos son una forma de organizar actividades que no pueden ser tratadas dentro de los límites operativos normales de la organización. Por lo tanto, los proyectos se usan a menudo como un medio de lograr el plan estratégico de la organización, bien sea con el equipo del proyecto de la organización o con un proveedor de servicios contratado.

De esta manera se reconoce la dirección de proyectos como la aplicación de conocimientos, habilidades, herramientas y técnicas, a las actividades de un proyecto para satisfacer los planes estratégicos de la organización; mediante la aplicación e integración de los procesos de dirección de inicio, planificación, ejecución, seguimiento y control, y cierre (PMBOK, 2004). Por otra parte Fernández (2007), asegura que la gestión de proyectos es la organización y supervisión de todos sus aspectos a fin de lograr los objetivos con los recursos disponibles lo que implica el uso de técnicas y conocimientos básicos.

Ahora bien, todo proyecto donde se busque desarrollar un nuevo producto o servicio, efectuar cambios en la estructura, entre otros, tiene como finalidad trazar un objetivo para luego concluirlo, en tal sentido, todo depende de la necesidad que tenga la organización en determinado momento para definir lo que desea desarrollar.

En este marco referencial, entran los proyectos de inversión de capital como un plan que se realiza para producir un bien o servicio, útil al ser humano o a la sociedad en general (Fernández, 2007). Es así como a partir de una idea, expectativa, deseo o sueño; tal vez difuso y poco específico, nace un proyecto de inversión que paso a paso se va esclareciendo y toma forma una vez que las ideas previas, luego de atravesar ciertos pasos o etapas, se plasman en un 
diagnóstico inicial y una prospección sistemática de los posibles resultados a obtener en el caso de llevar adelante la decisión analizada.

Para Petróleos de Venezuela, los proyectos de inversión de capital son llevados a cabo a través de las guías de gerencia para proyectos de Inversión de Capital (GGPIC, 1999); esta guía contiene los lineamientos prácticos para la ejecución de un proyecto de manera normalizada $y$ ordenada, de modo que ningún detalle y/o paso importante se escape. De igual manera las GGPIC aseguran que, en la práctica, se defina el ciclo de vida de un proyecto en cinco (05) fases: visualización, conceptualización, definición, implantación y operación.

En los últimos años la Industria petrolera en la Costa Oriental del Lago, se ha visto afectada en el desarrollo de las diferentes etapas de proyectos de inversión de capital, al adquirir un gran número de equipos sin conocer el sitio o lugar de instalación y sin realizar el correspondiente estudio previo que permite conocer la factibilidad técnica y/o económica de su instalación, lo que genera enormes pérdidas monetarias en este sector ya que los materiales y/o equipos quedan en espera de nuevas evaluaciones, que nunca llegan, y luego caen en deterioro y obsolescencia.

Es así como, muy a pesar que la industria petrolera cuenta con los departamentos necesarios para el estudio de dichos proyectos, en los últimos años ha invertido grandes cantidades de dinero sin obtener el retorno de capital esperado; esta problemática da lugar a la siguiente pregunta de investigación ¿Cómo es la Gestión de Proyectos de Inversión de Capital en la Industria Petrolera de la Costa Oriental del Lago del estado Zulia?

\section{MÉTODO}

De acuerdo a los objetivos planteados, esta investigación fue considerada de tipo descriptiva, bajo un diseño de campo, transeccional. La población estuvo conformada por ocho (08) Gerencias de Infraestructura y Proceso, pertenecientes a la industria Petrolera del estado Zulia; las cuales presentan características comunes debido a que cumplen la misma función pero en áreas diferentes a lo largo del estado.

Para recopilar información se utilizó la técnica de la encuesta y el instrumento utilizado fue un cuestionario de medición de frecuencia. Para calcular la confiabilidad de dicho instrumento, se aplicó el instrumento como prueba piloto a ocho (08) personas con las características de la población; los resultados se calcularon mediante el coeficiente Alfa Cronbach a través de la herramienta Excel, de esta manera, luego de realizar el cálculo antes mencionado el resultado de la confiabilidad fue de 0,90; dicho valor al ser comparado con la escala de magnitud representa que el instrumento tiene una muy alta confiabilidad.

Una vez obtenidos los resultados se organizaron agrupándolos por dimensión y así efectuar análisis de la información, a través del baremo interpretación mostrado en tabla 1, para desarrollar las conclusiones y recomendaciones de la investigación. 
Tabla 1. Baremo de interpretación

\begin{tabular}{cccc}
\hline $\begin{array}{c}\text { Escala de } \\
\text { Medición }\end{array}$ & $\begin{array}{c}\text { Rango de valores } \\
\text { para la media }\end{array}$ & Alternativas & Evaluación de la Gestión \\
\hline 3 & $2,26-3$ & Siempre & Muy Alto Cumplimiento \\
2 & $1,6-2,25$ & Casi Siempre & Moderado Cumplimiento \\
1 & $0,76-1,5$ & Casi Nunca & Bajo Cumplimiento \\
0 & $0-0,75$ & Nunca & Muy bajo Cumplimiento \\
\hline
\end{tabular}

RESULTADOS Y DISCUSIÓN

Tabla 2. Estadística de la dimensión Visualización de la gestión de proyectos

\begin{tabular}{|c|c|c|c|c|c|c|}
\hline \multirow{2}{*}{$\begin{array}{c}\text { INDICADOR } \\
\text { ALTERNATIVAS }\end{array}$} & \multicolumn{2}{|c|}{ Objetivos del Proyecto } & \multicolumn{2}{|c|}{ Alcance del Proyecto } & \multicolumn{2}{|c|}{ Estimado de Costos } \\
\hline & FA & FP (\%) & FA & FP (\%) & FA & FP (\%) \\
\hline SIEMPRE & 14 & 58,33 & 10 & 41,67 & 10 & 41,67 \\
\hline CASI SIEMPRE & 2 & 8,33 & 12 & 50 & 9 & 37,50 \\
\hline CASI NUNCA & 4 & 16,67 & 2 & 8,33 & 4 & 16,67 \\
\hline NUNCA & 4 & 16,67 & 0 & 0 & 1 & 4,17 \\
\hline TOTAL & 24 & 100 & 24 & 100 & 24 & 100 \\
\hline X Indicador & \multicolumn{2}{|c|}{1,81} & \multicolumn{2}{|c|}{2,44} & \multicolumn{2}{|c|}{2,17} \\
\hline X Dimensión & \multicolumn{6}{|c|}{2,14} \\
\hline
\end{tabular}

La tabla 2, muestra los resultados obtenidos para la dimensión visualización la cual presenta una media de 2,14 el cual al ser comparado con el baremo de interpretación, refleja un moderado cumplimiento, lo que justifica los problemas que se presentan a lo largo del proyecto ya que evidencia que no se establece una base sólida al comienzo del proceso productivo, lo que origina desviaciones en la finalidad del proyecto y a su vez incertidumbre en los costos (bajos o sobre estimados. Asimismo los resultados de la dimensión visualización ponen de manifiesto debilidades para canalizar los objetivos, al inicio del proceso, en correspondencia con las necesidades básicas del proyecto.

Este resultado se contrapone a lo expuesto por PMBOK (2004), cuando expresa la necesidad de que en esta fase se documenten las necesidades, se establezcan descripciones claras de los objetivos del proyecto y su alcance, para finalmente hacer un pronóstico de los recursos para el análisis de inversión de la organización.

La tabla 3 muestra los resultados obtenidos para la dimensión conceptualización en la gestión de proyectos., la cual presenta una media de 2,09 (moderado cumplimiento). Teniendo en 
cuenta que ésta según PMBOK (2004) es la fase donde se recoge información de varias fuentes con diversos grados de completitud y confianza para desarrollar el plan de gestión del proyecto y asegurar su cabal cumplimiento; los resultados de los indicadores en su mayoría de cumplimiento moderado, indican oposición a lo expresado por el autor y evidencian problemas significativos en la manera de abordar el proyecto, por ejemplo es muy posible que el personal asignado no tenga las capacidades y/o destrezas necesarias en el área a trabajar. De igual manera estos resultados permiten predecir cierto desfase con los objetivos propuestos al comienzo del proyecto a ejecutar, así como que el proyecto se desarrolle en sitio y tiempo inadecuado generando retrasos y costos adicionales, entre otros.

Tabla 3. Estadística de la dimensión Conceptualización en la gestión de proyectos

\begin{tabular}{|c|c|c|c|c|c|c|c|c|}
\hline \multirow{2}{*}{$\begin{array}{c}\text { INDICADOR } \\
\text { ALTERNATIVAS }\end{array}$} & \multicolumn{2}{|c|}{$\begin{array}{c}\text { Definición del } \\
\text { equipo de Trabajo }\end{array}$} & \multicolumn{2}{|c|}{$\begin{array}{c}\text { Cronograma de } \\
\text { Trabajo }\end{array}$} & \multicolumn{2}{|c|}{$\begin{array}{l}\text { Definición de } \\
\text { Actividades }\end{array}$} & \multicolumn{2}{|c|}{ Localización } \\
\hline & FA & FP (\%) & FA & FP (\%) & FA & FP (\%) & FA & FP (\%) \\
\hline SIEMPRE & 4 & 25 & 9 & 37,50 & 6 & 37,50 & 1 & 6,25 \\
\hline CASI SIEMPRE & 5 & 31,25 & 13 & 50 & 8 & 50 & 12 & 75 \\
\hline CASI NUNCA & 6 & 37,50 & 2 & 12,50 & 2 & 12,50 & 3 & 18,75 \\
\hline NUNCA & 1 & 6,25 & 0 & 0 & 0 & 0 & 0 & 0 \\
\hline TOTAL & 16 & 100 & 24 & 100 & 16 & 100 & 16 & 100 \\
\hline X Indicador & \multicolumn{2}{|c|}{1,75} & \multicolumn{2}{|c|}{2,29} & \multicolumn{2}{|c|}{2,25} & \multicolumn{2}{|c|}{2,06} \\
\hline X Dimensión & \multicolumn{8}{|c|}{2,09} \\
\hline
\end{tabular}

Con respecto a los resultados de la dimensión definición en la gestión de proyectos de inversión de capital la tabla 4 muestra una media de 1,92 que al ser comparado con el baremo de interpretación, refleja un moderado cumplimiento.

Esto permite predecir, deficiencias en esta importante fase, lo que requiere mayor atención por parte de las gerencias encargadas de desarrollar los proyectos en la industria petrolera, con la finalidad de obtener resultados que contribuyan con el mejoramiento en los procesos; evitando así inconvenientes con los equipos, procesos, trabajos deficientes por la selección incorrecta del contratista y riesgos inherentes a las etapas a desarrollar. 
Estos resultados pasan por alto lo planteado por PMBOK (2004) cuando asegura que el riesgo de un proyecto es un evento o condición incierto que, si se produce, tiene un efecto positivo o negativo sobre al menos un objetivo del proyecto, como tiempo, costo, alcance o calidad; por lo que se debe realizar un proceso de gestión de riesgos del proyecto en sus distintas fases, que van desde la planificación hasta el seguimiento y control de todos los riesgos asociados al trabajo.
Se visualizan oportunidades de mejora para toda la dimensión lo que requiere realizar procesos de planificación, aseguramiento tecnológico y control de la calidad en todos los proyectos. De igual manera contactar licencias de tecnología que ayuden a tomar correctas decisiones en el área tecnológica, para mejorar el trabajo y acortar tiempos de entrega.

Tabla 4. Estadística de la dimensión Definición en la gestión de proyectos

\begin{tabular}{|c|c|c|c|c|c|c|c|c|}
\hline \multirow{2}{*}{$\begin{array}{c}\text { INDICADOR } \\
\text { ALTERNATIVAS }\end{array}$} & \multicolumn{2}{|c|}{ Riesgo } & \multicolumn{2}{|c|}{$\begin{array}{c}\text { Aseguramiento } \\
\text { Tecnológico }\end{array}$} & \multicolumn{2}{|c|}{$\begin{array}{c}\text { Aseguramiento de } \\
\text { Calidad }\end{array}$} & \multicolumn{2}{|c|}{$\begin{array}{l}\text { Selección de } \\
\text { Contratista }\end{array}$} \\
\hline & FA & FP (\%) & FA & FP (\%) & FA & $\mathrm{FP}(\%)$ & FA & FP (\%) \\
\hline SIEMPRE & 7 & 29,17 & 12 & 50 & 1 & 4,17 & 16 & 50 \\
\hline CASI SIEMPRE & 8 & 33,33 & 7 & 29,17 & 10 & 41,67 & 7 & 21,88 \\
\hline CASI NUNCA & 7 & 29,17 & 4 & 16,67 & 12 & 50 & 7 & 21,88 \\
\hline NUNCA & 2 & 8,33 & 1 & 4,17 & 1 & 4,17 & 2 & 6,25 \\
\hline TOTAL & 24 & 100 & 24 & 100 & 24 & 100 & 32 & 100 \\
\hline X Indicador & & & & & & & & \\
\hline X Dimensión & & & & & 1,92 & & & \\
\hline
\end{tabular}

En relación con los resultados de la dimensión Implantación en la Gestión de Proyectos, la tabla 5 muestra una media de 2.50 que al ser comparado con el baremo de interpretación, refleja un muy alto cumplimiento.

Los resultados alcanzados, permiten afirmar que las gerencias de infraestructura y proceso pertenecientes a la industria petrolera de la costa oriental del lago, mantienen muy alto cumplimiento en todos los indicadores relacionados a la implantación y control del proyecto, observándose oportunidades de mejora en el indicador relacionado con la procura de materiales el cual obtuvo resultado de moderado cumplimiento. 
El resultado de este indicador evidencia la presencia de ciertos inconvenientes en la procura de materiales y quipos, que incide sobre el stock de materiales, tiempo de ejecución y costos; lo que contrasta con lo expresado por PDVSA (1999), al afirmar que la procura de materiales es una actividad primordial para lograr la ejecución del proyecto dentro del tiempo y calidad planificados; para lo que es necesaria la coordinación de programas de seguimiento, control y aseguramiento de calidad de los materiales, y asegurar que la compra de materiales y equipos se haga de acuerdo con las listas de proveedores aprobados.

Tabla 5. Estadística de la dimensión Implantación en la gestión de proyectos

\begin{tabular}{|c|c|c|c|c|c|c|c|c|}
\hline \multirow{2}{*}{$\begin{array}{c}\text { INDICADOR } \\
\text { ALTERNATIVAS }\end{array}$} & \multicolumn{2}{|c|}{$\begin{array}{c}\text { Revisión y firma de } \\
\text { Contrato }\end{array}$} & \multicolumn{2}{|c|}{$\begin{array}{l}\text { Administración de } \\
\text { Contrato }\end{array}$} & \multicolumn{2}{|c|}{$\begin{array}{l}\text { Procura de } \\
\text { materiales y } \\
\text { equipos }\end{array}$} & \multicolumn{2}{|c|}{$\begin{array}{c}\text { Control de variables } \\
\text { del proyecto }\end{array}$} \\
\hline & FA & FP (\%) & FA & FP (\%) & FA & FP (\%) & FA & FP (\%) \\
\hline SIEMPRE & 23 & 95,83 & 16 & 66,67 & 8 & 28,13 & 16 & 66,67 \\
\hline CASI SIEMPRE & 1 & 4,17 & 6 & 25 & 12 & 37,50 & 8 & 33,33 \\
\hline CASI NUNCA & 0 & 0 & 2 & 8,33 & 6 & 18,75 & 0 & 0 \\
\hline NUNCA & 0 & 0 & 0 & 0 & 5 & 15,63 & 0 & 0 \\
\hline TOTAL & 24 & 100 & 24 & 100 & 32 & 100 & 24 & 10 \\
\hline X Indicador & \multicolumn{2}{|c|}{2,96} & \multicolumn{2}{|c|}{2,58} & \multicolumn{2}{|c|}{1,78} & \multicolumn{2}{|c|}{2,67} \\
\hline X Dimensión & \multicolumn{8}{|c|}{2,50} \\
\hline
\end{tabular}

En relación con los resultados de la dimensión Operación en la Gestión de Proyectos., la tabla 6 muestra una media de 2.20 que al ser comparada con el baremo de interpretación, refleja un moderado cumplimiento, con resultados de muy alto cumplimiento para los indicadores puesta en marcha, y cierre financiero lo que evidencia la atención y cuidado que las gerencias en estudio dispensan a estos aspectos, utilizando políticas definidas de la puesta en marcha y sobre cierre financiero de los proyectos realizados para asegurar la puesta en marcha definitiva.
El resultado de estos indicadores confirma lo expuesto por PDVSA (1999) en donde la finalización de una obra es un proceso gradual donde se terminan de construir diferentes elementos de la planta que deben ser revisados y acondicionados para el arranque; buscando alcanzar el nivel mínimo para la operación estable, probar el desempeño de los equipos, verificar la calidad de los productos, entre otros aspectos. 
Se aprecian oportunidades de mejora para el resto de los indicadores que presentaron resultados de moderado cumplimiento, para su alineación con lo expuesto por PDVSA (1999) al sugerir que luego de un periodo razonable se proceda a llevar a cabo pruebas de rendimiento a la capacidad de la instalación durante un periodo previamente acordado para verificar su capacidad y calidad, corregir problemas al momento de entrega e inconvenientes que surjan por evaluaciones deficientes.

Tabla 6. Estadística de la dimensión Operación en la gestión de proyectos

\begin{tabular}{|c|c|c|c|c|c|c|c|c|c|c|}
\hline \multirow{2}{*}{$\begin{array}{l}\text { INDICADOR } \\
\text { ALTERNATIVAS }\end{array}$} & \multicolumn{2}{|c|}{$\begin{array}{l}\text { Puesta en } \\
\text { Marcha }\end{array}$} & \multicolumn{2}{|c|}{$\begin{array}{l}\text { Pruebas de } \\
\text { Garantía }\end{array}$} & \multicolumn{2}{|c|}{ Cierre Físico } & \multicolumn{2}{|c|}{$\begin{array}{c}\text { Cierre } \\
\text { Financiero }\end{array}$} & \multicolumn{2}{|c|}{$\begin{array}{c}\text { Evaluación } \\
\text { continua }\end{array}$} \\
\hline & FA & FP (\%) & FA & FP (\%) & FA & FP (\%) & FA & FP (\%) & FA & FP (\%) \\
\hline SIEMPRE & 8 & 50 & 8 & 33,33 & 7 & 29,17 & 15 & 62,50 & 15 & 50 \\
\hline CASI SIEMPRE & 8 & 50 & 6 & 25 & 2 & 50 & 8 & 33,33 & 8 & 25 \\
\hline CASI NUNCA & 0 & 0 & 7 & 29,17 & 1 & 4,17 & 0 & 0 & 0 & 25 \\
\hline NUNCA & 0 & 0 & 3 & 12,50 & 4 & 16,67 & 1 & 4,17 & 1 & 0 \\
\hline TOTAL & 16 & 100 & 24 & 100 & 24 & 100 & 24 & 100 & 24 & 100 \\
\hline X Indicador & \multicolumn{2}{|c|}{2,50} & \multicolumn{2}{|c|}{1,79} & \multicolumn{2}{|c|}{1,92} & \multicolumn{2}{|c|}{2,54} & \multicolumn{2}{|c|}{2,25} \\
\hline X Dimensión & \multicolumn{10}{|c|}{2,20} \\
\hline
\end{tabular}

En relación con los resultados de la variable gestión de proyectos, la tabla 7 muestra una media de 2.17 que al ser comparada con el baremo de interpretación, refleja un moderado cumplimiento, de las políticas utilizadas para dar respuesta a los proyectos de inversión de capital realizados en la industria petrolera.

Los resultados permiten inferir que los proyectos cumplen moderadamente con los lineamientos expresados en las (Guías de gerencia para proyectos de inversión de capital (GGPIC), comúnmente utilizadas en la industria petrolera para dar curso a los proyectos. Resalta como el aspecto más débil, la fase de definición, lo que deja abierta la posibilidad de nuevas investigaciones que permitan visualizar oportunidades de mejoras en esta dimensión que es base para el inicio, desarrollo y cierre de un proyecto. 
Tabla 7. Estadística de la variable gestión de proyectos

\begin{tabular}{ccc}
\hline DIMENSIÓN & X DIMENSIÓN & X VARIABLE \\
\hline VISUALIZACIÓN & 2,14 & \\
CONCEPTUALIZACIÓN & 2,09 & $\mathbf{2 , 1 7}$ \\
DEFINICIÓN & 1,92 & \\
IMPLANTACIÓN & 2,50 & \\
OPERACIÓN & 2,20 & \\
\hline
\end{tabular}

\section{Discusión}

Para Griffin (1993) (citado por Turner y col., 2003), la gestión es un conjunto de actividades (entre las que se incluyen planificación y toma de decisiones, organización, liderazgo y control), dirigidas a los recursos de una organización (humanos, financieros, físicos e informativos) con el fin de alcanzar de modo eficaz, los objetivos de la misma.

En este mismo orden de ideas, la Norma ISO 9000:2005, define la gestión como actividades coordinadas para dirigir $y$ controlar una organización; por otra parte Griful (2005), define gestión como el correcto aprovechamiento de los recursos disponibles y el uso de procedimientos adecuados para cumplir con los objetivos planteados.

En lo expresado por Griffin, Norma ISO 9000 y Griful, se encuentra coincidencia en que la gestión se orienta a la planificación de actividades coordinadas con el fin de alcanzar los objetivos planteados por la organización de una manera eficaz y dar así forma y cabida al proyecto a ejecutar.

En el marco de este contexto, un proyecto según PMBOK (2004) es un esfuerzo temporal que se lleva a cabo para crear un producto, servicio o resultado único; el final se alcanza cuando se han logrado los objetivos del proyecto o cuando queda claro que los objetivos del proyecto no serán o no podrán ser alcanzados; o cuando la necesidad del proyecto ya no exista y el proyecto sea cancelado. Por otra parte, Cartay (1996) afirma que todo proyecto consiste de un conjunto ordenado de acciones que atienden a la realización de un determinado fin. Sea sencillo o complejo, todo proyecto tiene un inicio y un fin definidos en el tiempo, y se conciben como una secuencia de actividades tendientes a buscar, analizar y coordinar un conjunto de informaciones y datos que justifiquen, según ciertos criterios, su ejecución.

Los autores PMBOK y Cartay, están de acuerdo que todo proyecto es un conjunto de acciones para crear un conjunto o servicio, el cual tiene un inicio y un fin definidos en el tiempo.

Desde las perspectivas de gestión y proyecto analizadas en los párrafos anteriores, se define gestión de proyectos según PMBOK (2004) como el conjunto de herramientas, técnicas, metodologías, recursos y procedimientos utilizados para gestionar un proyecto. Puede ser formal o 
informal, y ayuda al director del proyecto a gestionarlo de forma eficaz hasta su conclusión.

Al respecto Vertice (2008), afirma que la gestión de proyectos es el proceso de planeación y manejo de tareas y recursos con el fin de cumplir con los objetivos definidos para la implementación de un proyecto nuevo en la empresa, y la comunicación permanente del progreso y avance de sus resultados.

En este mismo orden de ideas Briceño (1995), define proyecto de inversión como el desarrollo de distintas fases, desde que se concibe la idea hasta su implementación final, pasando por todas sus etapas intermedias. Asimismo, Fernández (2007), afirma que un proyecto de inversión es una propuesta que surge como resultado de estudios que la sustentan y que está conformada por un conjunto determinado de acciones con el fin de lograr ciertos objetivos.

En este sentido, Briceño y Fernández concuerdan que todo proyecto de inversión presenta un conjunto determinado de acciones o etapas desde la idea inicial hasta su implementación final.

Es importante mencionar que un proyecto de inversión de capital debe tener un estudio previo a su creación, puesto que debe estar sustentado técnica y económicamente. Se debe de igual manera, conceptualizar y evaluar la rentabilidad de su puesta en marcha.

Para PDVSA (1999), en la gestión de proyectos es de vital importancia mantener un proceso y una metodología estandarizada y aprobada para ejecutar las tareas de desarrollo del mismo desde su concepción hasta su materialización y puesta en marcha; por tal motivo expone que todo proyecto para su cristalización debe seguir una serie de fases tales como: visualización, conceptualización, definición, implantación y operación.

De igual manera PMBOK (2004), establece como primera fase la etapa de iniciación en donde se componen los procesos que facilitan la autorización formal para comenzar un nuevo proyecto o una fase del mismo; antes de comenzar con las actividades del grupo de procesos de iniciación, se documentan las necesidades o requisitos de negocio de la organización, se establecen descripciones claras de los objetivos del proyecto, incluidas las razones por las cuales un proyecto específico es la mejor solución alternativa para satisfacer los requisitos. La documentación de esta decisión también contiene una descripción básica del alcance del proyecto, de los productos entregables, de la duración del proyecto y un pronóstico de los recursos para el análisis de inversión de la organización.

Para el autor, en los proyectos de múltiples fases, los procesos de iniciación se llevan a cabo en fases posteriores para validar las asunciones realizadas y las decisiones tomadas durante los procesos originales, desarrollar el acta de constitución del proyecto y desarrollar el enunciado del alcance del proyecto (preliminar). Además, durante el proceso de iniciación se refina la descripción del alcance inicial y los recursos que la organización está dispuesta a invertir. Esta información se refleja en el Acta de Constitución del Proyecto $y$, una vez aprobado, el proyecto queda oficialmente autorizado.

De igual manera, dentro del marco PDVSA (1999), las GGPIC, establecen para la etapa de visualización, una serie de elementos necesarios para la consolidación, tales como: objetivos del proyecto, alcance del proyecto y estimados de costo; todos de gran importancia en esta fase. 
En cuanto a los objetivos, Miranda (2005) expresa que son metas a corto, mediano y largo plazo que se esperan alcanzar. Todo proyecto debe tenerlos claramente definidos en términos cuantitativos y cualitativos, de forma tal que los responsables puedan utilizar instrumentos de medición para confrontar las metas propuestas con las realmente alcanzadas y aplicar correctivos en caso de desviaciones.

Para Cartay (1996), un objetivo es una etapa preestablecida que conduce a una finalidad precisa, por tanto, se define cuando se ha fijado el sujeto de la acción, se ha cuantificado la finalidad y se ha determinado el plazo para alcanzarla.

Al contrastar lo expresado por Miranda y Cartay, se evidencia que dichos autores coinciden en que los objetivos son metas con plazos definidos, por otra parte PDVSA enfoca, que los objetivos planteados en todo proyecto deben satisfacer los objetivos corporativos de la empresa.

Luego que los objetivos y propósitos del proyecto se establecen, y los grupos de planificación constatan que cumplen con las estrategias y lineamientos del plan de negocios, se debe elaborar un alcance preliminar, como base para estimar costo y tiempo de ejecución (PDVSA, 1999). Estos estimados se utilizan en el análisis para confirmar la factibilidad económica del proyecto y la conveniencia de proseguir con su desarrollo. La elaboración de este alcance preliminar debe ser un trabajo de equipo, a fin de contar con la experiencia e información tanto del dueño como de la organización de ingeniería y proyectos.

Por otra parte para PMBOK (2004), el alcance del proyecto es el proceso necesario para producir una definición preliminar de alto nivel, usando el Acta de Constitución con otras entradas a los procesos de iniciación. Los autores PDVSA y PMBOK, coinciden en que el Alcance del Proyecto da inicio a la conformación de lo que realmente se quiere realizar mediante objetivos ya definidos, donde se documenta todo el proceso para evitar introducir elementos adicionales que distorsionen el Alcance definitivo.

En relación al estimado de costos, PDVSA (1999) afirma que se expresan con precisión los precios, en orden de magnitud, los cuales se utilizan en la planificación a mediano plazo para establecer si los proyectos reúnen los méritos suficientes para proseguir su desarrollo. Es importante mencionar que, los métodos de estimación se basan en datos históricos de costos que provienen de proyectos similares ejecutados o curvas de unidades de proceso similares (extrapolación estadística), correlacionadas por su capacidad y corregidas por índices de precios, factores de ubicación geográfica, entre otros.

Adicionalmente PMBOK (2004), aporta que la estimación de costes de las actividades del cronograma implica desarrollar una aproximación de los costes de los recursos necesarios para completar cada actividad del cronograma. Por otra parte esta incluye la identificación y consideración de diversas alternativas de costes y evalúa si las reducciones de costes esperadas pueden compensar el coste del trabajo adicional de diseño.

En cuanto a la fase de conceptualización, PDVSA (1999), define que el propósito es la selección de la(s) mejor(es) opción(es) y la mejora en la precisión de los estimados de costos y tiempo de implantación. Todo esto para lograr reducir la incertidumbre $y$ cuantificar los riesgos asociados y determinar el valor esperado para la(s) opción(es) seleccionada(s) con la finalidad de cumplir con los siguientes objetivos: 
organizar para la fase de planificación del proyecto, seleccionar la(s) opción(es) preferida(s) y solicitar los fondos para ejecutar las actividades que permitan obtener un estimado de costo Clase II.

En relación con este aspecto, PMBOK (2004), establece la conceptualización como la segunda fase del grupo de procesos de planificación donde se recoge información de varias fuentes con diversos grados de completitud y confianza. En la planificación se desarrolla el plan de gestión del proyecto; igualmente se identifica, define y madura su alcance, el coste y actividades a desarrollar.

Dentro de ese marco PDVSA (1999), establece en las GGPIC, para la etapa de conceptualización, una serie de elementos que buscan dar respuesta a las necesidades presentes en esta fase: Definición del equipo de trabajo: Esta se efectúa con base a la participación organizacional / funcional y requiere atributos individuales de sus miembros, experiencia - conocimiento cabal de los elementos clave del proyecto, capacidad - la habilidad de ejecutar las tareas requeridas por el proyecto $y$ autoridad - la capacidad de tomar decisiones; para el logro de sus objetivos. Todas las organizaciones involucradas deben tener representación en el equipo y tener oportunidad de aportar al proceso de planificación.

Por otra parte Miranda (2005), expone que la formación del equipo de trabajo de un proyecto consiste en examinar la gestión de la diversidad de sus miembros, donde la primera condición para su integración y adaptación es voluntariedad, se requiere su motivación de querer ser miembro del equipo. En segundo lugar, se deben estudiar las competencias necesarias para pertenecer al equipo: profesionales, sociales y psicológicas. Por último, además de poseer las características anteriores, una persona para trabajar en equipo debe tener una formación específica en el trabajo a desarrollar.

Cabe destacar que para PMBOK (2004), adquirir el equipo del proyecto es el proceso de obtener los recursos humanos necesarios para completar el trabajo. El equipo de dirección puede o no tener control sobre los miembros del equipo seleccionados para el proyecto.

Los miembros del equipo se obtienen de todas las fuentes disponibles, tanto internas como externas, por lo tanto una o más de las organizaciones que participan en el proyecto pueden tener políticas, guías o procedimientos que rigen las asignaciones de personal; los departamentos de recursos humanos también pueden ayudar en el reclutamiento, la contratación y la orientación de los miembros del equipo del proyecto.

Otro de los elementos de la fase de conceptualización es el cronograma de trabajo. EI PMBOK (2004), afirma que su desarrollo es un proceso iterativo, que determina fecha de inicio y finalización de las actividades del proyecto y exige que se revisen y corrijan las estimaciones de duración y de los recursos, para crear un cronograma aprobado del proyecto que sirva como línea base con respecto a la cual medir el avance. El desarrollo del cronograma se mantiene a lo largo del proyecto, a medida que el trabajo avanza.

Al respecto Miranda (2005), expone que al culminar los diferentes estudios técnicos se está en capacidad de elaborar un "cronograma de actividades" donde con alguna precisión se señalan las principales actividades con sus respectivas fechas de realización.

En lo expresado por PMBOK y Miranda, se observa similitud puesto que ambos afirman que el cronograma de trabajo no es 
más que un proceso que orienta su finalidad a la planificación de tareas con fechas de inicio y finalización, aunado a las características específicas de acuerdo a las actividades a realizar.

En cuanto al proceso de definición de las actividades de esta fase, expresa PMBOK (2004) que el proceso de definir las actividades del cronograma, implica identificar y documentar el trabajo que se planifica realizar; en él se identifican los productos entregables al nivel más bajo de la estructura de desglose del trabajo (EDT) para proporcionar una base que permita estimar y establecer el cronograma, así como ejecutar, supervisar y controlar el trabajo del proyecto de tal modo que se cumplan sus objetivos.

Al mismo tiempo Briceño (1995), sostiene que la programación de las actividades debe visualizarse como un proceso iterativo y de sucesivos afinamientos, en la medida que progresa el desarrollo de la ingeniería, mejora la estimación de ubicaciones, se asignan los primeros contratos, y se confirman los equipos principales. Paralelamente, se abren las etapas en subetapas, las actividades en subactividades y se mejora la definición y exactitud de las secuencias y rendimientos.

Al contrastar lo expresado por, PMBOK y Briceño se percibe que ambos autores afirman que el cronograma de actividades es el proceso que se realiza para documentar lo que se desea realizar en el proyecto, aunado a que se busca el rendimiento y exactitud de todas sus etapas.

\section{CONCLUSIONES}

Con relación a la dimensión visualización los resultados indican un moderado cumplimiento en la primera fase de los proyectos en cuanto a la realización de los objetivos y la estimación de costos; mientras que en la elaboración del alcance de proyecto los resultados arrojan un muy alto cumplimiento. Estos resultados ponen de manifiesto que habrá problemas a los largo del proyecto ya que no se concibe un alcance de proyecto que no concuerde con los objetivos y los estimados de costo, la falta de una base sólida como comienzo del proceso productivo, facilita desviaciones en la finalidad del proyecto y a su vez costos bajos o sobre estimados

Por otra parte, con respecto a la dimensión conceptualización que obtuvo en los indicadores: Definición del equipo, de actividades y localización, moderado cumplimiento; mientras el cronograma de trabajo alcanzó un muy alto cumplimiento, y al comparar la media de la dimensión con el baremo de interpretación la ubica en moderado cumplimiento, lo que indica que el proyecto presenta inconvenientes al momento de contratar personal calificado de acuerdo al área de estudio, de igual manera al caracterizar las actividades de mayor peso e importancia y por ultimo con decisiones erradas en cuanto al sitio e a ejecutar el proyecto en cuestión.

Asimismo, con relación a la dimensión operación los resultados demuestran que su cumplimiento es moderado lo que significa que los proyectos realizados en la industria petrolera mantienen deficiencia en la consideración de los riesgos asociados al proceso, así como en el aseguramiento de la calidad de los equipos o materiales utilizados y también en la manera de realizar la selección del contratista; lo que podría conllevar a realizar operaciones con alto riesgo, equipos con baja calidad de servicio y a su vez instalaciones finales no acordes a lo planteado al inicio del proyecto ejecutado.

Con respecto a la dimensión implantación, los resultados indican a nivel general un alto cumplimiento, lo que permite inferir que la industria petrolera a 
través de las gerencias de infraestructura y proceso, manejan un alto número de contrataciones dentro de las normativas para tal fin, Se enfatiza en la necesidad del correcto manejo de la procura de materiales y equipos para evitar futuros gastos adicionales y mantener los niveles de stock ideales para reducir tiempos muertos en el proceso.

En cuanto a la dimensión operación, los resultados obtenidos demuestran un moderado cumplimiento en la etapa final del proyecto, lo que podría suscitar problemas con la documentación final presentada por ambas partes, en donde se describen todos los procesos realizados con las actividades, tiempos de entrega y costos asociados, así como también con la baja presencia de pruebas de garantía que demuestren previamente el correcto funcionamiento de los equipos o la instalación.

Finalmente se concluye, que existe un moderado cumplimiento en la gestión de proyectos de inversión en la industria petrolera, lo que puede ocasionar problemas en los proyectos y generar altos costos adicionales a lo estimado, tiempo excesivo en la entrega de las instalaciones, personal no acorde con los requerimientos del trabajo a ejecutar, baja calidad en los procesos productivos, entre otros.

\section{REFERENCIAS}

Briceño, P. (1995). El Desarrollo de Proyectos. Editorial Mc Graw Hill. Chile

Cartay, I. (1996). Planificación y Control de Proyectos. Maracaibo - Venezuela

Fernández, S. (2007). Los Proyectos de Inversión. Editorial Tecnológica de Costa Rica. Primera edición. Costa Rica

Griful, E. y col (2005). Gestión de la Calidad. Primera Edición. Ediciones UPC. Barcelona

ISO 9000: 2005 (2005). Sistema de Gestión de la Calidad. Conceptos y vocabulario

Miranda, J. (2005). Gestión de Proyectos. MM Editores. Quinta Edición. Bogotá Colombia

PMBOK. (2004). Guía de los Fundamentos de la Dirección de Proyectos. Project Management Institute. Tercera Edición. Philadelphia - Estados Unidos

PDVSA (1999). Guías de Gerencia para Proyectos de Inversión de Capital. PDVSA. Caracas - Venezuela

Rubio, P. (2008). Introducción a la Gestión Empresarial. Instituto Europeo de Gestión Empresarial. Madrid - España

Turner, A. y col (2003). Terapia Ocupacional y Disfunción Física. Principios, Técnicas y Prácticas. Editorial Elsevier Science. Quinta Edición. Madrid. España 